\title{
Redox conformation changes in refined tuna cytochrome $c$
}

(x-ray diffraction/electron transfer mechanism/protein conformation changes/buried water molecule/heme motion in protein)

\section{TSUNEHIRO TAKANO AND RICHARD E. DICKERSON}

Norman W. Church Laboratory of Chemical Biology, California Institute of Technology, Pasadena, California 91125

Communicated by Emanuel Margoliash, July 21, 1980

\begin{abstract}
Tuna ferrocytochrome $c$ and ferricytochrome $c$ have been refined independently at high resolution $(1.5 \AA$ and $1.8 \AA$ ) to crystallographic residual errors of $17.3 \%$ and $20.8 \%$, respectively. Small but significant conformational differences are seen surrounding a buried water molecule that is hydrogen bonded to Asn-52, Tyr-67, and Thr-78. In the oxidized state, this water molecule is $1.0 \AA$ closer to the heme and the heme has moved $0.15 \AA$ out of its heme crevice; both changes lead to a more polar microenvironment for the heme. Chemical modification studies, patterns of evolutionary conservatism, structural differences in bacterial cytochromes, and $x$-ray studies all agree that the "active site" for cytochrome $c$ is bounded by lysines 8 , $13,27,72,79,86$, and 87 (thus containing the evolutionary conservative 72-87 loop) and has the buried water molecule just below its surface and the opening of the heme crevice slightly to one side.
\end{abstract}

Respiratory cytochrome $c$ from mitochondria is perhaps the best-known and most intensively studied of all electron-transport proteins-from the spectroscopic studies of MacMunn in 1887 (1) through the virtual rediscovery of the protein by Keilin in 1925 (2) and work by Theorell and the Stockholm school (3) to the many investigations in other laboratories (4-6). Its near omnipresence in oxygen-respiring life forms, ease of isolation and purification, and relative stability under in vitro conditions have made it an ideal candidate for examination of electrontransfer processes between macromolecules. The same properties have also made it especially suitable for study by $x$-ray diffraction methods. Our investigations were begun at Caltech in 1963 in collaboration with E. Margoliash; they have resulted in a 4- $\AA$ resolution map of horse ferricytochrome $c$ in 1967 (7), a 2.8- $\AA$ resolution map that revealed the polypeptide chain path (8), a $2.45-\AA$ analysis of tuna ferrocytochrome $c$ (9), and structure analyses of both ferricytochrome $c$ and ferrocytochrome $c$ from tuna at $2.0 \AA$ that included optimized modelbuilding and fitting of idealized bond parameters to the resultant electron density map (10-12). In parallel with this work, the Osaka protein structure group has produced a $2.3-\AA$ analysis of bonito ferrocytochrome $c(13-15)$ and a $2.8-\AA$ analysis of the ferric form (16). None of the above work, however, has resulted in a molecular structure that is truly refined in the crystallographic sense, and it has been impossible to determine what changes, if any, occurred in the molecule as it shifted from one heme oxidation state to the other.

This paper describes the results of high-resolution crystallographic refinement of tuna ferricytochrome $c$ and ferrocytochrome $c$ that has led to standard crystallographic residual errors ( $R$ factors) of $17.3 \%$ for ferrocytochrome $c$ at 1.5 - $\AA$ resolution and $20.8 \%$ for ferricytochrome $c$ at $1.8-\AA$ resolution.

The publication costs of this article were defrayed in part by page charge payment. This article must therefore be hereby marked "advertisement" in accordance with 18 U. S. C. $\$ 1734$ solely to indicate this fact.

\section{METHODS}

Tuna cytochrome $c$ was extracted, purified, and crystallized as described $(9,10)$. X-ray data were collected at room temperature on a Syntex $\mathbf{P} \overline{1}$ automatic diffractometer to $1.5-\AA$ resolution for ferrocytochrome $c$ (space group $\mathrm{P} 22_{1} 2{ }_{1} 2$; cell dimensions of $a=37.33 \AA, b=87.10 \AA, c=34.44 \AA$ ) and to $1.8-\AA$ resolution for ferricytochrome $c$ (space group $\mathrm{P4}_{3}$; cell dimensions of $a=b=74.42 \AA, c=36.30 \AA$ ). Merging of new data with the earlier $2-\AA$ sets and rejection of intensities below $2 \sigma$ gave 13,840 statistically significant intensities for ferrocytochrome $c$ refinement and 16,831 for ferricytochrome $c$.

The refinement process is described in more detail elsewhere (unpublished data). It essentially consisted of Diamond model building and real-space refinement (17-19), followed by Jack-Levitt energy refinement (20). At present, ferrocytochrome $c$ has an $R$ factor of $17.3 \%$ and 52 solvent molecules, and ferricytochrome $c$ has an $R$ factor of $20.8 \%$ and 49 solvent molecules. Luzzati's method of estimating root-mean-square errors in atomic positions (21) gives $0.15 \AA$ for the reduced molecule and $0.20 \AA$ for the oxidized. For a rigid ring or other group of $n$ atoms, this can be reduced by a factor of $\sqrt{n}$, making reasonable estimates for errors within a pyrrole ring of $\approx 0.07 \AA$ and $\approx 0.09 \AA$, respectively. Twelve solvent molecules were found at the same relative positions in all three molecules; three of these are discussed in detail in this paper.

\section{RESULTS}

Three cytochrome $c$ molecules were refined in this analysis: the ferrocytochrome molecule (designated reduced or simply $R$ ) and the two crystallographically independent ferricytochrome molecules in the oxidized crystal. The latter two will be differentiated by designating the molecule involved in the $4_{3}$ screw axis through the origin of the cell as the outer molecule and that around the screw axis through the center of the cell as the inner molecule. Refined coordinates for all three molecules have been deposited with the Brookhaven Protein Data Bank (Long Island, NY), replacing the 1976 coordinates.

This refinement at high resolution has led to differences betweeen ferrocytochrome $c$ and ferricytochrome $c$ that are believable because they are departures from the starting coordinates and are greater than the refined differences between the two independent ferricytochrome molecules. Comparision of the root-mean-square differences in corresponding atom positions in all three pairs of molecules shows that the differences between oxidation states are approximately $0.10 \AA$ greater than those between outer and inner molecules for all classes of atoms - main chain, side chains, and heme. (For a graphical description of a detailed atom-by-atom comparison of distances between corresponding atoms in the three molecules and a discussion of its significance, see ref. 22.)

The conformational changes with redox state that we believe to be most significant are best shown by comparison of their 


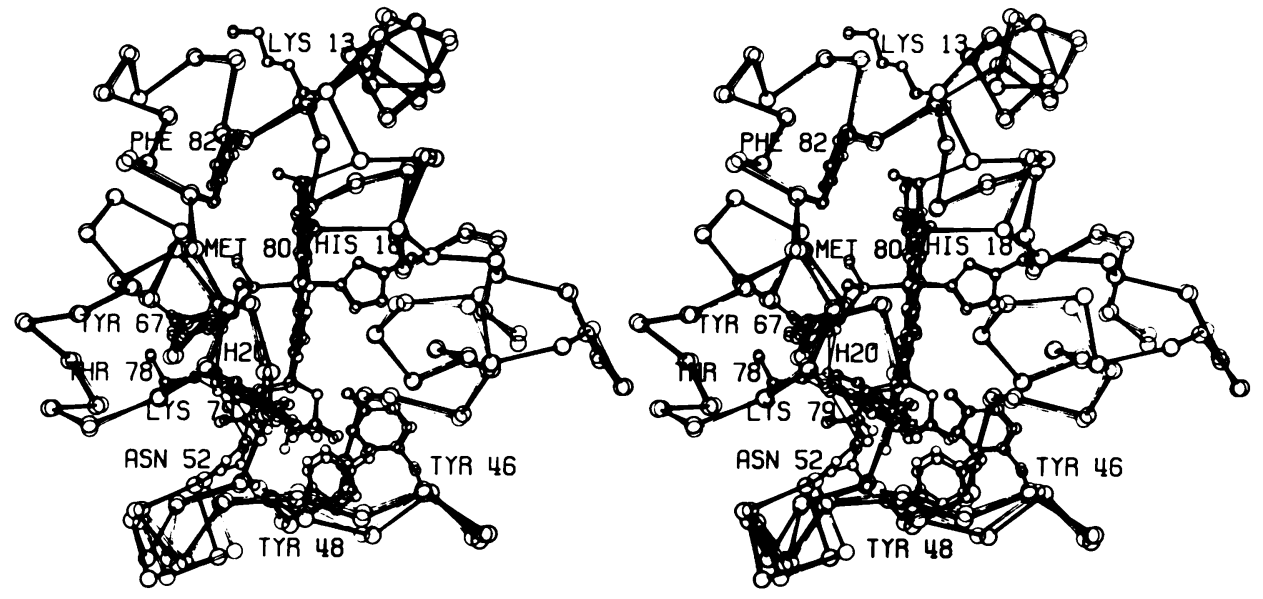

FIG. 1. Superimposed stereo-pair drawings of reduced or ferrocytochrome $c$ molecule (solid bonds) and inner ferricytochrome $c$ molecule (open bonds). Aside from minor differences around residues 27 and 28 that arise from intermolecular packing contacts in the oxidized crystal, the two redox states differ principally at the lower portion of the molecule, in the vicinity of Asn-52 (see Fig. 2).

stereo drawings (Figs. 1 and 2) with Fig. 3 as a control. On reduction, the heme group moves $0.15 \AA$ into the crevice, roughly along a vector connecting pyrrole rings 3 and 1 . $^{*}$ A more precise description would be to say that it pivots around the methyl side chain of ring 2. Regions in which the redox conformational differences are significantly greater than the differences between ferricytochrome molecules include Cys-17 to Thr-19 (the heme attachment), Lys-27 to Leu-35 (the lower right lining of the heme crevice), Lys-39 to Ala-44, and Ser-47 to Asn-60 (the floor of the crevice), and groups Tyr-67, Met-80, Phe-82, and Ala-83 (all of which are directly involved with the heme).

The most striking changes are those that occur in the network of hydrogen bonds around the water molecule buried to the left of the heme crevice. These changes are shown accurately in Figs. 1 and 2 and schematically in Fig. 4. As the heme sinks into the crevice, the hydrogen bond between its buried propionate and Trp-59 drags that group $0.4 \AA$ toward the interior of the molecule. Tyr-48, also bonded to that propionate, is pushed downward. The buried water molecule sinks $1.0 \AA$ toward the

* Pyrrole ring numbering: 1 = buried propionate, 2 = Cys-14 attachment, 3 = Cys-17 attachment, $4=$ outer propionate. This agrees with current numbering practice of the Brookhaven Protein Data Bank and publications on other heme proteins. In some earlier papers, our rings $\mathbf{1 - 4}$ are numbered IV, I, II, and III, respectively. bottom of the molecule. Its hydrogen-bonded Asn-52 side chain twists, and the backbone of the " 50 s" helix moves down and to the left (Fig. 1). The hydrogen bond between Asn-52 and the main chain amide of residue 41 decreases from $3.5 \AA$ to $3.0 \AA$ and that between the water molecule and Tyr- 67 pulls that group $0.7 \AA$ down and into the interior. A third hydrogen bond from the water molecule extends to the hydroxyl group of Thr-78, which seems to act as a motionless pivot during reduction.

The geometry of the heme group and the interaction between the group and ligands in the three molecules are summarized in Table 1. The iron-nitrogen distances to His-18 and the iron-sulfur distances are consistent with those observed in model compounds (23-25). In neither the model compounds nor in tuna cytochrome is a change in oxidation state of the iron accompanied by significant changes in $\mathrm{Fe}-\mathrm{N}$ and $\mathrm{Fe}-\mathrm{S}$ bond lengths.

\section{DISCUSSION}

The differences between the conformations of reduced and oxidized cytochrome $c$ proved in the end to be smaller than had been expected, or had been believed at intermediate stages of the structure analysis, and to lie in a region of the molecule whose importance to mechanism had not previously been
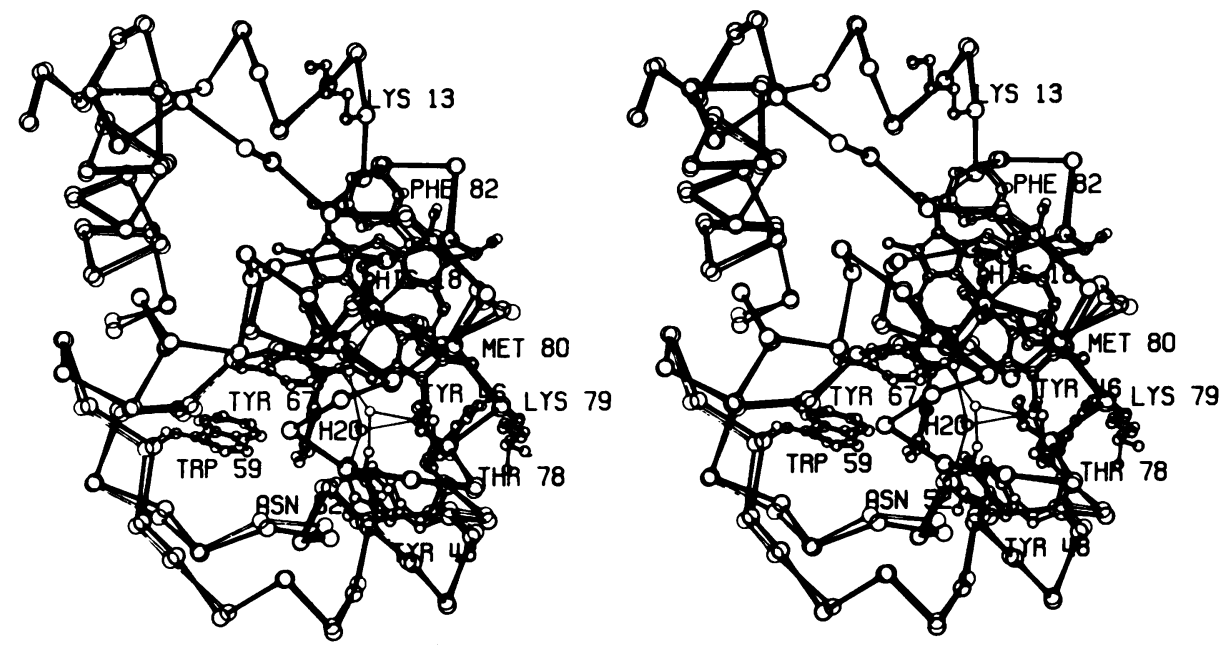

FIG. 2. Left side view of the reduced (solid bonds) and inner (open bonds) molecules. 

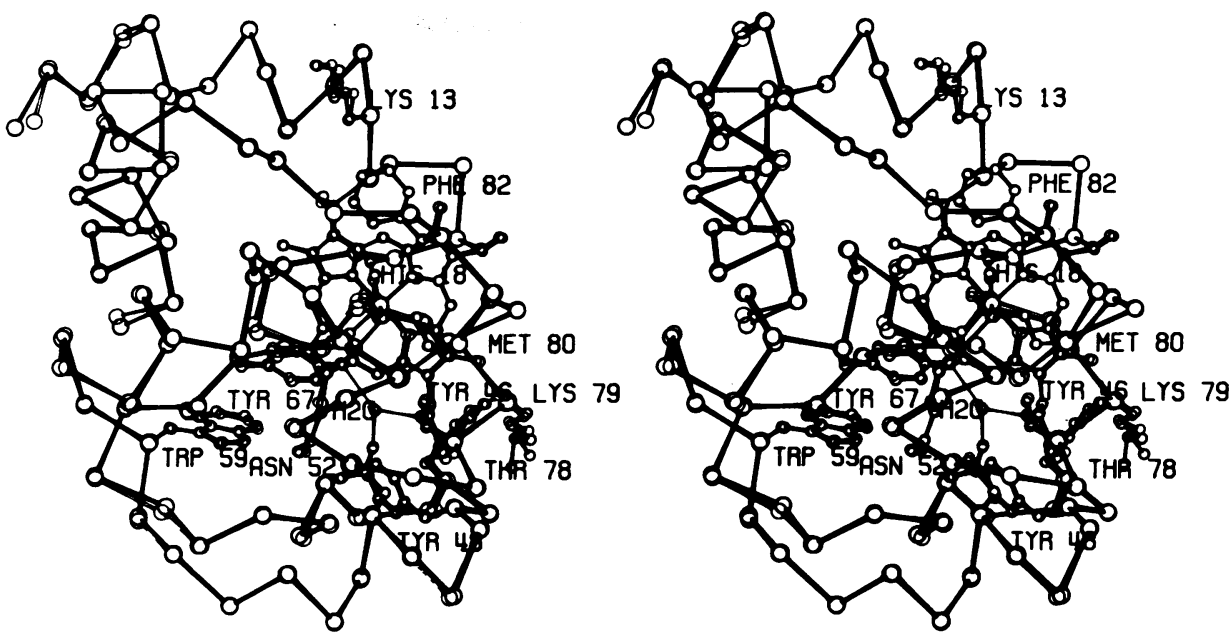

FiG. 3. The "control" for Figs. 1 and 2: a superposition from the same orientation of the two ferricytochrome $c$ molecules, outer (solid bonds) and inner (open bonds). There are no important differences except at the amino and carboxy termini (which also have large temperature factors); in particular, there are none of the changes around the water molecule.

considered-the bottom left side of the heme crevice. The shift in Asn-52 with redox change was actually observed in reoxidized-minus-reduced difference maps in August 1972, but its significance was discounted at the time (11). In retrospect, these early observations give us confidence in the accuracy of the present refinement.

The buried water molecule that is hydrogen bonded to Asn-52, Thr-78, and Tyr-67 lies just under the molecular surface as defined by the 70s loop of polypeptide chain, which had been noticed to be evolutionarily conservative at the very beginning of sequence and structure analysis $(8,26)$. In the light of recent chemical modification and protection studies of the interaction of cytochrome $c$ with its oxidase, reductase, and peroxidase, this region of the molecular surface assumes more importance as possibly being the contact surface with the larger macromolecules between which it transfers electrons. Millet and Margoliash have independently prepared series of cytochrome $c$ derivatives in which a single lysine side chain is modified, and then they looked for altered reactivity of each derivative with mitochondrial cytochrome oxidase (27-29) and reductase (30,31),

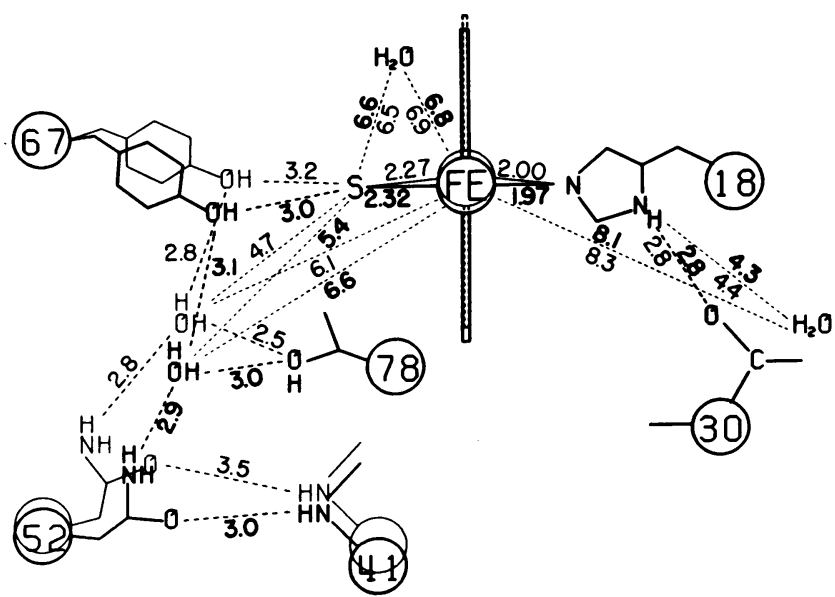

FIG. 4. Schematic drawing of side-chain motion in the vicinity of the heme. Heavy lines indicate the reduced molecule, and light lines indicate the oxidized molecule. Hydrogen bonds are dashed; bond distances between nonhydrogen atoms are in Ångstrom units. Distances for the oxidized state are averages of the outer and inner molecules. The three water molecules shown schematically here are shown accurately in Fig. 5. yeast cytochrome $c$ peroxidase (32), and liver microsomal cytochrome $b_{5}(33)$. The latter is relevant because it has been shown to resemble a core domain of yeast cytochrome $b_{2}$-lactate dehydrogenase, for which cytochrome $c$ is an in vivo electron acceptor (34). In a somewhat different but complementary approach, Bosshard and Pettigrew have studied the shielding of lysines against chemical modification that is produced by binding cytochrome $c$ to its oxidase $(35,36)$, reductase $(35,37)$, or peroxidase (38). All of these studies define the same binding site on the cytochrome $c$ molecule: the upper front of the heme crevice, roughly centered around Phe- 82 and bounded by lysines $8,13,27,72,79,86$, and 87 and to a lesser extent $5,7,25$, 73 , and 88 . In contrast, lysines $22,39,53,55,60,99$, and 100 at the bottom and rear of the molecule are not involved in interactions with any of the electron donor or acceptor macromolecules.

The "active site" of cytochrome $c$, as defined by these lysine

Table 1. Heme geometry

\begin{tabular}{|c|c|c|c|}
\hline & Reduced & Outer & Inner \\
\hline \multicolumn{4}{|c|}{ Distance, $\AA$} \\
\hline Fe to heme plane* & 0.06 & 0.01 & 0.04 \\
\hline Fe to NNNN plane ${ }^{\dagger}$ & 0.07 & 0.03 & 0.01 \\
\hline Fe to pyrrole $\mathrm{N}$ (avg.) & 2.06 & 2.08 & 2.03 \\
\hline Fe to $\mathrm{N} \epsilon$ of His-18 & 1.97 & 1.96 & 2.04 \\
\hline Fe to $\mathrm{S} \delta$ of Met- 80 & 2.32 & 2.28 & 2.26 \\
\hline N $\epsilon$ of His-18 to NNNN plane & 1.90 & 1.97 & 2.03 \\
\hline $\mathrm{S} \delta$ of Met-80 to NNNN plane & 2.39 & 2.23 & 2.20 \\
\hline \multicolumn{4}{|c|}{ Angle, ${ }^{\circ}$, between heme-plane normal and } \\
\hline Pyrrole 1-plane normal & 5.1 & 6.3 & 3.2 \\
\hline Pyrrole 2-plane normal & 11.1 & 10.8 & 5.6 \\
\hline Pyrrole 3-plane normal & 14.2 & 10.1 & 8.9 \\
\hline Pyrrole 4-plane normal & 7.8 & 5.5 & 4.6 \\
\hline NNNN-plane normal & 3.8 & 2.3 & 3.7 \\
\hline $\mathrm{Fe}-\mathrm{N}$ bond to His-18 & 2.7 & 5.2 & 4.9 \\
\hline $\mathrm{Fe}-\mathrm{S}$ bond to Met- 80 & 5.7 & 9.9 & 10.4 \\
\hline \multicolumn{4}{|c|}{ Angle, ${ }^{\circ}$, between NNNN-plane normal and } \\
\hline $\mathrm{Fe}-\mathrm{N}$ bond to His-18 & 2.8 & 6.5 & 8.2 \\
\hline $\mathrm{Fe}-\mathrm{S}$ bond to Met- 80 & 2.4 & 9.6 & 12.3 \\
\hline \multicolumn{4}{|c|}{ Angle, ${ }^{\circ}$, between imidazole ring plane normal and } \\
\hline $1 \mathrm{~N}-3 \mathrm{~N}$ vector & 47 & 51 & 52 \\
\hline
\end{tabular}

* Defined by porphyrin core plus first attached carbon atoms on perimeter.

$\uparrow$ Defined by four pyrrole nitrogen atoms. 
modification studies, is shown in Fig. 5. The 70s portion of main chain loops through this region, and the buried, hydrogenbonded water molecule lies just beneath the surface. Two other external water molecules are found in the vicinity of the heme crevice in both oxidation states: one between the heme ring 3-ring 4 methylene and the Ile-81 side chain and the other close to the hydrogen bond between the $\mathrm{N}_{\delta}$ of His-18 and the main chain $\mathrm{CO}$ of Pro-39 (i.e., within hydrogen-bonding distances of the NH of Thr-19, the CO of Lys-25, the NH of His-26, and the CO of Gly-29).

In addition to lysine modification studies, several other lines of evidence suggest that regions of the cytochrome surface other than the face shown in Fig. 5 are unimportant in binding to electron-transferring macromolecules. Cytochromes reconstituted from incomplete chains, in which excess or loose chain segments have been trimmed away by trypsin, have been found to exhibit normal spectral properties and be reducible by cytochrome $b_{2}$-lactate dehydrogenase, even though they have a chain break between residues 53 and 54 or are missing chain segments 26-27 and 39-55 entirely (39-42). Indeed, not only is the lower part of the molecule apparently unnecessary, it even seems to unfold easily once the chain is broken. Trypsin will attack reconstituted cytochrome $c$ that has only one defect-a chain break after residue 38-and digest away residues 39-55 but preserve $46 \%$ of the residual activity with $b_{2}$-lactate dehydrogenase (41). Finally, the resistance to or acceptance of amino acid substitutions among 89 known eukaryotic cytochromes $c$ helps to delineate the critical vs. the unimportant regions of the molecule. The 24 evolutionarily invariant positions $(1,6,10,17,18,30,32,34,38,41,48,59,68,70,71,73$, $76-80,82,84$, and 91 ) line the heme crevice, ringing the active site (as defined in Fig. 5) and making up the 70s loop that weaves through this site. In contrast, the 13 most variable positions, where 8-11 different amino acids are found $(3,12,33$, $54,58,60-62,65,88,89,92$, and 100 ), occur without exception on the top, back, and bottom of the molecule. Thus, Fig. 5 may be a very good representation of the view of cytochrome $c$ that is presented as it approaches its oxidase, reductase, peroxidase, or cytochrome $b_{2}$-lactate dehydrogenase.

The 70s loop sequence-

71

Pro-Lys-Lys-Tyr-Ile-Pro-Gly-Thr79

Lys-Met-Ile-Phe-Ala-Gly-Ile-Lys-Lys-Lys

is remarkable in that those side chains facing the outside (see Fig. 5) (shown in boldface) are uniformly either basic or hydrophobic, as if this surface had evolved to fit against another having a central hydrophobic region surrounded by a ring of negative charges. Exactly this surface topography has been observed by Poulos and Kraut in their recent $\mathrm{x}$-ray analysis of cytochrome $c$ peroxidase (43), which shows an excellent spatial match between lysines $13,27,72,86$, and 87 on cytochrome $c$ and the aspartic acid side chains $37,39,216,37$, and 34 on the peroxidase. Similar complementarity might be expected on the surfaces of oxidase and reductase.

The region of chain that is easily unfolded and removed by trypsin, residues 39-55, is just that region at the bottom of the molecule that shows the greatest difference between reduced and oxidized states (Figs. 1 and 2) and corresponds to the region that is deleted in the short S-class cytochromes $c$ such as Pseudomonas $c_{551}$, Chlorobium $c_{555}$, and Anacystis $c_{554}(44,45)$. Motion of this bottom loop of the molecule may explain the apparent easy accessibility of the region around Tyr-67 and the buried water molecule to external reagents such as $\mathrm{KI}_{3}(46)$ and tetranitromethane $(47,48)$. Evidently, there is enough internal molecular adjustment to allow entry of reagent molecules but not enough to permit rotation of the tyrosine ring. Thus, although Tyr-48 appears to be buried and hydrogen bonded to an extent similar to Tyr-67, its unreactivity implies that this part of the molecule is not flexible enough to permit access by $\mathrm{KI}_{3}$ or $\mathrm{C}\left(\mathrm{NO}_{2}\right)_{4}$.
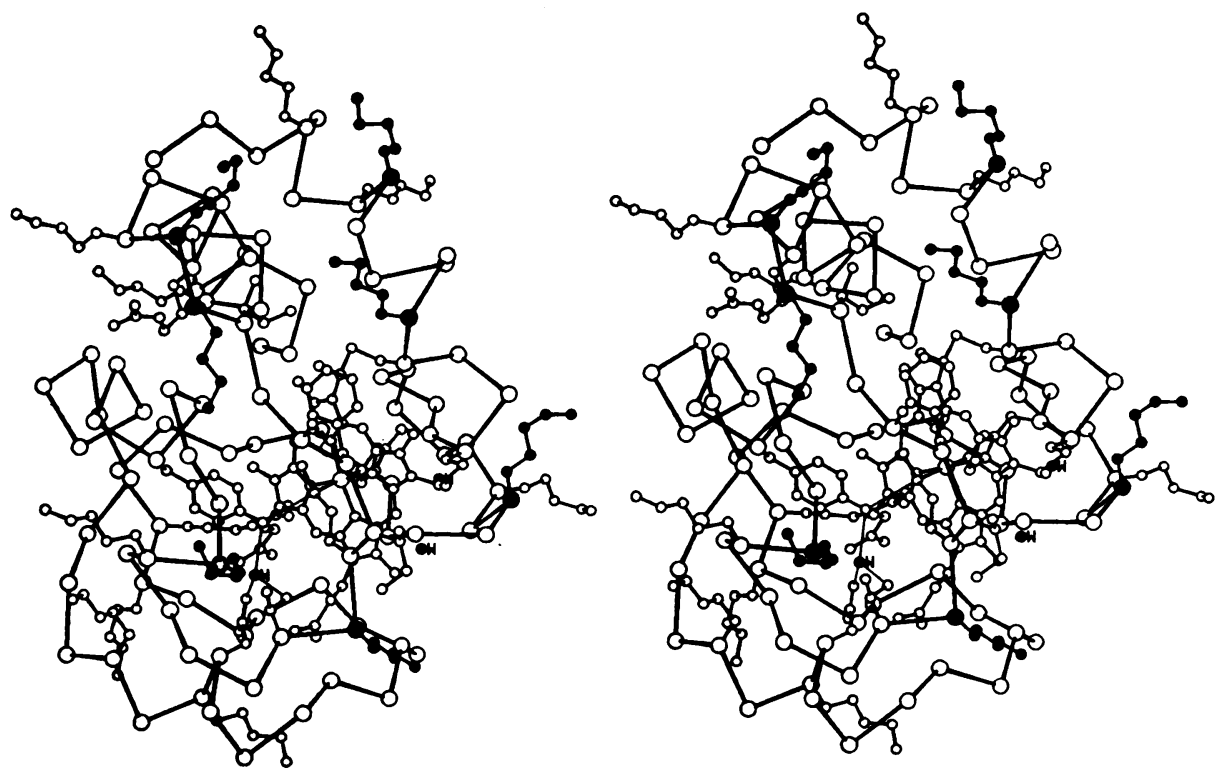

FIG. 5. View into the "active site" of reduced tuna cytochrome $c$, as defined by the seven lysines (black side chains) that have been shown to participate in binding to cytochrome oxidase, reductase, and peroxidase. Reading clockwise around the active site from the upper right, these are lysines $8,13,27,79,72,86$, and 87 . The other lysines (unblackened side chains) are either secondarily involved $(5,7,25,73$, and 88$)$ or definitely not involved $(22,39,53,55,60,99$, and 100$)$ in binding to other redox macromolecules. These lysines occur on the perimeter or the back side of the molecule as viewed here. Phe-82 sits at the center of the ring of positive charge around the active site; the heme crevice opens slightly to the right. The evolutionarily conservative polypeptide chain from Lys-72 to Lys-87 snakes across the face of this region, which may be the contact surface with other electron-transferring macromolecules. W, The three bound water molecules observed in both redox states. The hydrogen bonds to Tyr-67, Thr-78, and Asn-52 surrounding the buried water molecule are just beneath the 70s loop. 
What contribution might the buried but moving water molecule and its network of hydrogen bonds make to the cytochrome $c$ mechanism? Its role might be a purely passive and structural one, helping to stabilize the left side of the heme crevice and shifting its position in response to the formal charge on the heme (ferroheme is neutral whereas ferriheme has a +1 charge). Both the $1.0-\AA$ shift of the buried water molecule toward the heme and the 0.15- $\AA$ movement of the heme out of its crevice would be natural responses to the increased polarity of the heme after oxidation. However, cause and effect are difficult to untangle; if, in some manner, the binding of ferrocytochrome $c$ to oxidase were to induce the shift of the water molecule, then the more polar microenvironment of the heme produced would assist the loss of an electron. The ferricytochrome molecule, making a better fit against the oxidase surface than ferrocytochrome, would then be expected to be a competitive inhibitor of further ferrocytochrome reaction, which is known to be true (49). Unfortunately for such an aesthetically pleasing hypothesis, little or no difference can be seen (Figs. 1 and 2) for that region of the cytochrome $c$ surface that is most likely to come in contact with other electron-transferring enzymes. At present, the water molecule can only be assigned a structural role, helping by its hydrogen bonds to hold together the left half of the molecule.

We thank George A. Carlson and Ben Conner for their help in various phases of crystal preparation, data collection, and data processing. This is Contribution No. 6231 from the Norman W. Church Laboratory of Chemical Biology, California Institute of Technology. This work was performed with the support of National Institutes of Health Grant GM-12121 and National Science Foundation Grant PCM 79-13959.

1. MacMunn, C. A. (1887) J. Physiol. 8, 51-56.

2. Keilin, D. (1966) The History of Cell Respiration and Cytochrome, (Cambridge Univ. Press, Cambridge, England).

3. Åkeson, Å. \& Ehrenberg, A., eds. (1972) Structure and Function of Oxidation-Reduction Enzymes (Pergamon, Oxford).

4. Margoliash, E. \& Schejter, A. (1966) Adv. Protein Chem. 21, 113-286.

5. Lemberg, R. \& Barrett, J. (1973) Cytochromes (Academic, New York).

6. Dickerson, R. E. \& Timkovich, R. (1975) in The Enzymes, ed. Boyer, P. D. (Academic, New York), 3rd Ed., Vol. 11, pp. 397-547.

7. Dickerson, R. E., Kopka, M. L., Weinzierl, J., Varnum, J., Eisenberg, D. \& Margoliash, E. (1967) J. Biol. Chem. 242, 3015-3017.

8. Dickerson, R. E., Takano, T., Eisenberg, D., Kallai, O. B., Samson, L., Cooper, A. \& Margoliash, E. (1971) J. Biol. Chem. 246, 1511-1535.

9. Takano, T., Kallai, O. B., Swanson, R. \& Dickerson, R. E. (1973) J. Biol. Chem. 248, 5234-5255.

10. Swanson, R., Trus, B. L., Mandel, N., Mandel, G., Kallai, O. B. \& Dickerson, R. E. (1977) J. Biol. Chem. 252, 759-775.

11. Takano, T., Trus, B. L., Mandel, N., Mandel, G., Kallai, O. B., Swanson, R. \& Dickerson, R. E. (1977) J. Biol. Chem. 252, 776-785.

12. Mandel, N., Mandel, G., Trus, B. L., Rosenberg, J., Carlson, G. \& Dickerson, R. E. (1977) J. Biol. Chem. 252, 4619-4636.

13. Ashida, T., Ueki, T., Tsukihara, T., Sugihara, A., Takano, T. \& Kakudo, M. (1971) J. Biochem. 70, 913-924.
14. Ashida, T., Tanaka, N., Yamane, T., Tsukihara, T. \& Kakudo, M. (1973) J. Biochem 73, 463-465.

15. Tanaka, N., Yamane, T., Tsukihara, T., Ashida, T. \& Kakudo, M. (1975) J. Biochem. 77, 147-162.

16. Matsuura, Y., Hata, Y., Yamaguchi, T., Tanaka, N. \& Kakudo, M. (1979) J. Biochem. 85, 729-737.

17. Diamond, R. (1966) Acta Crystallogr. 21, 235-266.

18. Diamond, R. (1971) Acta Crystallogr. Sect. A 27, 436-452.

19. Diamond, R. (1974) J. Mol. Biol. 82, 371-391.

20. Jack, A. \& Levitt, M. (1978) Acta Crystallogr. Sect. A 34, 931-935.

21. Luzatti, V. (1952) Acta Crystallogr. 5, 802-810.

22. Takano, T. \& Dickerson, R. E. (1980) in Interaction Between Iron and Proteins in Oxygen and Electron Transport, ed. Ho, C. (Elsevier/North Holland, New York), in press.

23. Bowman, K., Gaughan, A. P. \& Dori, Z. (1972) J. Am. Chem. Soc. 94, 727-721.

24. Collins, D. M., Countryman, R. \& Hoard, J. L. (1972) J. Am. Chem. Soc. 94, 2066-2072.

25. Mashiko, T., Marchon, J.-C., Musser, D. T., Reed, C. A., Kastner, M. E. \& Scheidt, W. R. (1979) J. Am. Chem. Soc. 101, 36533655

26. Margoliash, E. (1963) Proc. Natl. Acad. Sci. USA 50, 672-679.

27. Staudenmayer, N., Ng, S., Smith, M. B. \& Millett, F. (1977) Biochemisty 16,600-604.

28. Smith, H. T., Staudenmayer, N. \& Millett, F. (1977) Biochemistry 16, 4971-4974.

29. Ferguson-Miller, S., Brautigan, D. L. \& Margoliash, E. (1978) J. Biol. Chem. 253, 149-159.

30. Ahmed, A. J., Smith, H. T., Smith, M. B. \& Millett, F. (1978) Biochemistry 17, 2479-2483.

31. Speck, S. H., Ferguson-Miller, S., Osheroff, N. \& Margoliash, E. (1979) Proc. Natl. Acad. Sci. USA 76, 155-159.

32. Kang, C. H., Brautigan, D. L., Osheroff, N. \& Margoliash, E. (1978) J. Biol. Chem. 253, 6502-6510.

33. Ng, S., Smith, M. B., Smith, H. T. \& Millett, F. (1977) Biochemistry 16, 4975-4978.

34. Guiard, B., Groudinsky, O. \& Lederer, F. (1974) Proc. Natl. Acad. Sci. USA 71, 2539-2543.

35. Rieder, R. \& Bosshard, H. R. (1978) FEBS Lett. 92, 223-226.

36. Rieder, R. \& Bosshard, H. R. (1978) J. Biol. Chem. 253, 60456053.

37. Bosshard, H. R., Zürrer, M., Schägger, H. \& von Jagow, G. (1979) Biochem. Biophys. Res. Commun. 89, 250-258.

38. Pettigrew, G. (1978) FEBS Lett. 86, 14-16.

39. Hantgan, R. R. \& Taniuchi, H. (1977) J. Biol. Chem. 252 , 1367-1374.

40. Hantgan, R. R. \& Taniuchi, H. (1978) J. Biol. Chem. 253, 5373-5380.

41. Parr, G. R., Hantgan, R. R. \& Taniuchi, H. (1978) J. Biol. Chem. 253, 5381-5388.

42. Juillerat, M., Parr, G. R. \& Taniuchi, H. (1980) J. Biol. Chem. 255, 845-853.

43. Poulos, T. L. \& Kraut, J. (1980) J. Biol. Chem., in press.

44. Dickerson, R. E. (1980) Sci. Am. 242 (3), 136-153.

45. Dickerson, R. E. (1980) Nature (London) 283, 210-212.

46. McGowan, E. B. \& Stellwagen, E. (1970) Biochemistry 9, 3047-3052.

47. Sokolovsky, M., Aviram, I. \& Schejter, A. (1970) Biochemistry 9, 5113-5118.

48. Schejter, A., Aviram, I. \& Sokolovsky, M. (1970) Biochemistry 9,5118-5122.

49. Yonetani, T. \& Ray, G. S. (1965) J. Biol. Chem. 240, 33923398 